\title{
Poison Center Data for Public Health Surveillance: Poison Center and Public Health Perspectives
}

\author{
Royal K. Law*1, Josh Schier ${ }^{1}$, Jay Schauben², Katherine Wheeler ${ }^{3}$ and Prakash Mulay ${ }^{4}$ \\ ${ }^{1}$ Centers for Disease Control and Prevention, Chamblee, GA, USA; ${ }^{2}$ Florida Poison Information Center - Jacksonville, Jacksonville, \\ FL, USA; ${ }^{3}$ New York City Department of Health and Mental Hygiene, New York City, NY, USA; ${ }^{4}$ Florida Department of Health, \\ Tallahassee, FL, USA
}

\section{Objective}

To describe the use of poison center data for public health surveillance from the poison center, local, state, and federal public health perspectives and to generate meaningful discussion on how to address the challenges to collaboration.

\section{Introduction}

Since 2008, poisoning has become the leading cause of injury-related death in the United States (US); since 1980, the poisoning-related fatality rate in the US has almost tripled. ${ }^{1}$ Many poison-related injuries and deaths are reported to regional poison centers (PCs) which receive about 2.4 million reports of human chemical and poison exposures annually. ${ }^{2}$ Federal, state, and local public health $(\mathrm{PH})$ agencies often collaborate with poison centers and use PC data for public health surveillance of poisoning-related health issues. Many state and local PH agencies have partnerships with regional PCs for direct access to local PC data which help them perform this function. At the national level, CDC conducts public health surveillance for exposures and illnesses of public health significance using the National Poison Data System (NPDS), the national PC reporting database.

Though most PC and PH officials agree that PC data play an important role in $\mathrm{PH}$ practice and surveillance, collaboration between PH agencies and PCs has been hindered by numerous challenges. To address these challenges and bolster collaboration, the Poison Center and Public Health Collaborations Community of Practice (CoP) was created in 2010 by CDC as a means to share experiences, identify best practices, and facilitate relationships among federal, state and local public health agencies and PCs. To date, the Poison Center and Public Health Collaborations CoP includes over 200 members from state and local public health, regional PCs, CDC, the American Association of Poison Control Centers (AAPCC), and the Environmental Protection Agency (EPA). A leadership team was created with representatives of the many stakeholders of the community to drive its direction and oversee activities.

\section{Methods}

The panel will consist of 4 presenters and 1 moderator, who are members of the Poison Center and Public Health Collaborations CoP leadership team. Each presenter will bring a unique perspective of the use of PC data for $\mathrm{PH}$ practice and surveillance: CDC, state department of health, a local department of health, and a PC. Royal Law from the CDC National Center for Environmental Health will present on using PC data for identification of exposures and illnesses of public health significance identified from NPDS data collected from all 57 PCs. Dr. Jay Schauben from the Florida/USVI Poison Information Center - Jacksonville will discuss PC participation in surveillance and use of $\mathrm{PC}$ data for tracking and mitigation of $\mathrm{PH}$ events in Florida. Dr. Prakash Mulay from the Florida Department of Health will discuss utilization of PC data to enhance ESSENCE-based chemical-associated exposure and illness surveillance in Florida. Katherine Wheeler from the New York City (NYC) Department of Health and Mental Hygiene will discuss NYC's use of PC data in surveillance of potential emerging issues, from energy drinks to synthetic marijuana. Each presenter will discuss the use of PC data for $\mathrm{PH}$ practice and surveillance in his or her organization and jurisdiction, the successes of using PC data, and their challenges.

\section{Results}

The moderator will engage the audience by facilitating discussion of the successes and challenges to using $\mathrm{PC}$ data for $\mathrm{PH}$ practice and surveillance with the audience.

Sample questions:

What are your current capacities and collaborative activities between your state/local health department and your poison center?

What non-funding related barriers hinder the collaboration between your state/local health department and poison center?

If more funding were available, how would you use this funding to increase the level of interactivity with the poison center and state/local health department?

\section{Keywords}

Surveillance; Poison Center; Community of practice

\section{References}

1. Warner M, Chen LH, Makuc DM, Anderson RN, and Minino AM Drug Poisoning Deaths in the United States, 1980-2008. National Center for Health Statistics Data Brief, December 2011. Accessed 8/29/2012.

2. Bronstein AC, Spyker DA, Cantilena LR, Green JL, Rumak BH, Dart RC. 2010 Annual Report of the American Association of Poison Control Centers' National Poison Data System (NPDS): 28th Annual Report. Clin Toxicol 2011; 49: 910-941.

\section{*Royal K. Law}

E-mail: hua1@cdc.gov 